Agnieszka Dryjańska

Université de Varsovie

Pologne

(iD https://orcid.org/0000-0003-1649-8408

\section{L'interculturel en classe de langue - analyse de corpus pour l'étude de différences et de ressemblances sémantiques du terme politesse en français et en polonais}

Intercultural approach in foreign language teaching corpus analysis in research of semantic differences and similarities of the word politeness in French and Polish

\begin{abstract}
This paper investigates an attempt to apply a corpus analysis to the development of the intercultural approach to foreign language teaching through the semantic analysis of cultural keywords. Our analysis will specifically include the process of discovering the meaning of the three words: French politesse and its Polish equivalents - grzeczność and uprzejmość through their collocations collected in French and Polish corpora. As a result, firstly, we came up with a conclusion that the French word politesse is characterized by more positive semantic prosody than its Polish equivalents. Secondly, politesse appears more frequently in coordinate collocations with nouns conveying aesthetical meaning, whereas the Polish word uprzejmośc is more frequently used with nouns designating ethical features.

The principles of our methodology or some data used in our research can inspire the introduction of an inductive lexical approach in foreign language teaching to facilitate the conceptualization of complex cultural notions, which is the essential of the intercultural teaching.
\end{abstract}

Keywords

Politeness, intercultural approach, culture, corpus analysis, semantic analysis 


\section{Introduction}

Chaque langue foisonne de mots chargés culturellement présentant un grand intérêt pour les littéraires, anthropologues, linguistes ou plutôt ethnolinguistes, mais aussi pour les didacticiens en langues étrangères, spécialement ceux qui privilégient l'approche interculturelle en classe de langue. Ce vocabulaire ne peut pas être traité de la même façon que tous les autres mots, la traduction constituant un danger particulièrement grave. En se contentant de fournir la traduction de mots comme liberté, patrie ou ami en polonais, anglais ou russe, on risque de transmettre une fausse image que ces mots ont le même sens dans toutes les langues. Rien de plus trompeur. L'ethnolinguiste polonaise Anna W ie r zbick a le confirme à chaque page de son ouvrage Understanding Cultures Through Their Key Words: English, Russian, Polish, German, and Japanese (1997). Chaque motclé cache une réalité différente, spécifique à une langue et culture.

Or, il est souvent difficile de comprendre les différences et les ressemblances sémantiques d'un mot chargé culturellement comme politesse, qui est l'objet de notre analyse, et encore plus difficile de les éclaircir en cours de langue. Comme matériel qui peut être exploité en classe, nous proposons une analyse de corpus qui véhiculent des informations sur la combinatoire lexicale correspondant au mot politesse en français et ses équivalents en polonais, pouvant être ensuite exploitées en classe. Notre démarche est inspirée de la méthodologie proposée par A. Wi e rzbicka (1997) qui se réfère entre autres à des corpus pour chercher des exemples concrets - passages de texte, phrases, expressions et mots qui révèlent le vrai sens de notions culturelles dans la perspective contrastive.

Il est à noter qu'il existe une littérature très riche abordant le phénomène de la politesse aussi bien en français qu'en polonais, souvent inspirée par des auteurs anglophones, éminents fondateurs des théories de la politesse et leurs principaux concepts comme face, territoire, self, actes menaçants (ang. FTAs) et actes flatteurs (ang. FFAs) (E. Goffma n, 2005 [1967] ; G. Le e ch, 1983 ; P. Brown, P. Levin son, 1987 ; R. Lak off, S. Ide, 2005 ; R. S collon, S. S collon, 1995). En l'occurrence, différents aspects de la politesse française ont été analysés dans la perspective sémantique, pragmatique, culturelle ou didactique (C. Kerbrat-Orecchioni, 1992, 1997, 2005, citée par C. Kerbrat-Orecchioni, 2010 ; O. Galatanu, 2010, O. Galatanu et A. Bellachhab, 2010 cités par P. C h a$\mathrm{raudeau}, 2014)$. Quant à la langue polonaise, il convient de citer au moins des chercheurs comme M. Marcjanik (1997), K. Ożóg (1990, 2001, 2014) et A. Żu rek (2008), qui se sont penchés sur le concept de politesse en général et sur la caractéristique de la politesse polonaise en particulier.

Toutes ces théories purement linguistiques ne contribuent guère à l'intégration des aspects variés de la politesse dans la perspective contrastive à la didactique des langues et cultures, vu que cette problématique occupe toujours une place 
très restreinte dans les manuels et les ressources didactiques traditionnelles. Les corpus eux-mêmes, les données et les outils de recherche qu'ils contiennent sont plus accessibles aux apprenants d'une langue étrangère pour plusieurs raisons. Tout d'abord, d'après mon expérience en tant qu'enseignante de français dans le cadre universitaire, l'apprenant d'aujourd'hui est habitué à des recherches par mots-clés sur Internet, qu'il effectue quotidiennement. La substitution du « corpus de Google » à un corpus professionnel serait donc un simple passage à une autre source de données n'entrainant aucun changement de paradigme de recherche. Ensuite, le recours à des conclusions fournies par des textes scientifiques portant sur la politesse est difficile, voire impossible, en pratique didactique en termes de niveau de langue et de connaissances variées indispensables à la compréhension des argumentations y exposées. Au contraire, l'exploration des données linguistiques contenues dans des corpus permettra à l'apprenant d'enrichir ses connaissances et de construire sa vision de certains phénomènes linguistico-culturels. Le potentiel didactique des corpus est d'autant plus grand qu'ils offrent de nombreux exemples d'usage et différents registres de langue.

\section{L'objectif de la recherche}

Nous proposons une démarche fondée sur l'analyse contrastive de la fréquence et des cooccurrences et des collocations adjectivales (et participiales) et coordonnées du nom politesse en français et ses équivalents polonais en tant qu'introduction à la reconstruction de la représentation linguistique de la réalité (RLR) de politesse dans ces deux langues afin de fournir des données et des méthodes favorisant le développement de l'approche interculturelle inductive en didactique du FLE. Au cœur de cette approche, il y a la prise de conscience de l'apprenant des convergences et divergences linguistico-culturelles qu'il découvre lui-même en analysant lesdites données. Notre hypothèse, fondée sur la fameuse constatation "You shall know a word by the company it keeps" et sur le fait qu'une partie du sens d'un mot est indiquée par ses collocations (J.R. Firth, 1962 [1957] : $11-12)$, est que les différences et les ressemblances sémantiques du mot politesse en français et en polonais se traduisent par différentes caractéristiques des cooccurrences, des collocations et de leur fréquence.

L'analyse est effectuée sur quatre corpus : le Corpus national de la langue polonaise (NKJP) version équilibrée qui compte (240 192461 mots), le corpus français Frantext - 253 millions de mots et les corpus sur le site Corpora.unileipzig (LC) qui offre des corpus en 252 langues, parmi lesquels celui en français (1 468766604 mots) et celui en polonais, beaucoup plus modeste (96 476260 mots).

En résumé, notre recherche s'inscrit dans deux domaines - la didactique des langues et cultures et la linguistique de corpus ; la première indiquant les objectifs de la recherche, la deuxième fournissant des données et des méthodes. 


\section{L'interculturel en FLE par le biais de la langue}

La notion clé de l'approche interculturelle en FLE est la compétence interculturelle, que nous définissons comme un ensemble de compétences qui se développent quand une culture étrangère se superpose sur celle de l'apprenant d'une langue étrangère et entraîne le changement de son attitude (A. Dryjańska, 2016). Le processus de développement de cette compétence est déclenché par le contact avec une langue étrangère, vecteur de culture. L'apprenant confronté aux différences et ressemblances culturelles reflétées par la combinatoire lexicale commence à s'interroger sur différents mécanismes langagiers, ce qui peut le mener vers une réflexion interculturelle. Le chemin de l'apprenant du contact avec une langue étrangère vers l'interculturel peut être représenté par le diagramme (fig. 1).

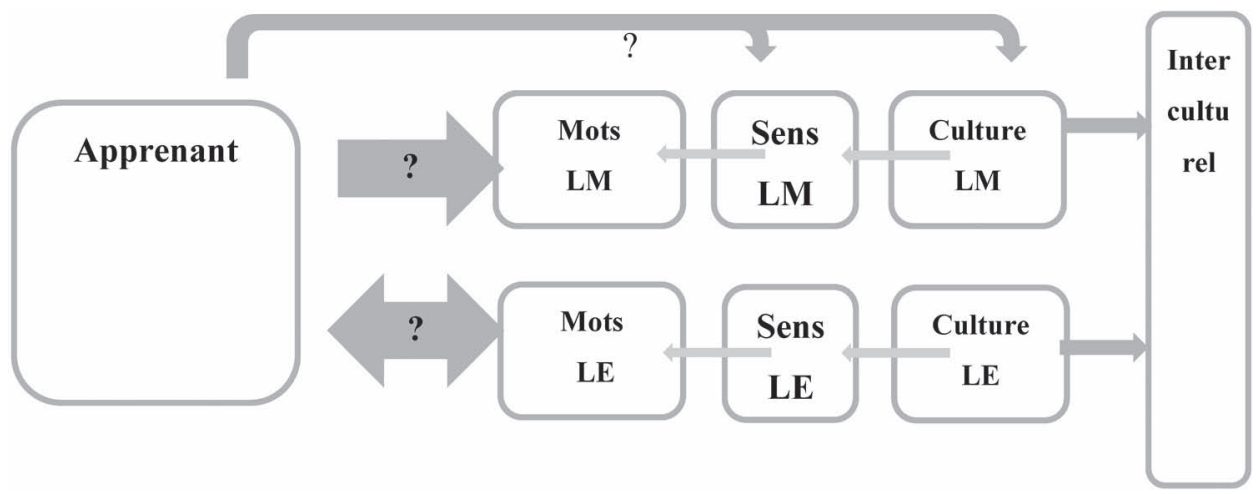

Fig. 1. Du mot vers l'interculturel. (Source : propre)

De très bons exemples d'approche fondée sur l'analyse de collocation sont proposés par S. Di Vito (2013), O. Tremblay (2015), O. Tremblay et D. A n ctil (2016) et M. Halliday (1966) cité par Ch. Mann ing, H. S chütze (1999). En analysant le contraste entre la collocation adjectivale strong tea (fr. thé fort, pl. mocna herbata) et la cooccurrence peu fréquente powerful tea (fr. thé puissant, pl. silna herbata), M. Halliday constate que "the contrast may tell us something interesting about attitudes towards different types of substances in our culture" (cité par Ch. Manning, H. S ch ütze, 1999 : 142) et il souligne que "it is obviously important to teach this contrast to students who want to learn idiomatically correct English" (1999 : 142). Nous sommes persuadés que la compréhension des attitudes envers des phénomènes culturels serait plus profonde si nous associions cette analyse à l'analyse des collocations adéquates en langue maternelle de l'apprenant. Si l'on abordait le mot tea, par exemple avec des élèves polonophones, il serait recommandé de s'appuyer sur un parallélisme étroit entre 
l'anglais et le polonais dans ce cas. Dans d'autres cas, l'enseignant devrait recourir à une analyse linguistique plus approfondie, p. ex. à une analyse de corpus.

Quant aux mots abstraits chargés culturellement, la situation en FLE est encore plus complexe que l'analyse du mot tea. L'appréhension du sens de ces mots est difficile pour l'apprenant qui ne connait pas le contexte social ni le contexte culturel très particulier qui a fait émerger ces termes. Dans la langue maternelle, le développement du langage chez l'enfant procède du concret à la généralisation, par contre, dans la langue étrangère, il y a un processus inverse (G. BaumgratzGangl, 1993 citée par A. Dry jań s k a, 2016). À son tour, R. Galisson, inventeur du terme lexiculture et grand partisan de l'intégration de ces " mots à charge culturelle partagée (CPP)»(R. Ga li s s o n, 1988 ; A. Fra n c e s c o, 2010) à la didactique des langues-cultures, souligne l'importance de la recherche du concret dans cette approche en parlant du rôle de la pragmatique lexiculturelle qui permet de mettre en œuvre « une procédure d'investigation allant du fait lexiculturel observé (concret) à l'idée qu'il y a derrière et le sous-tend (l'abstrait) » (R. Ga 1 is s o n, 2005). Pour que l'apprenant comprenne un mot étranger qui représente un concept complexe et abstrait comme la politesse, il doit découvrir les réalités concrètes qui l'ont fondée. Cela est faisable grâce à l'analyse du phénomène de la politesse en français et en polonais par le biais des collocations de ce mot. Nous proposons l'analyse de corpus et ses outils en tant que piste d'investigation lexiculturelle.

\section{Extraction des collocations dans un corpus}

Le corpus, parmi tous ses emplois variés, permet d'extraire des cooccurrences très fréquentes et des collocations qui, à notre sens, présentent un grand intérêt pour la didactique du FLE, surtout dans le cadre de l'enseignement de la « lexiculture ». Des chercheurs observent qu'« il paraît logique que les lexèmes les plus fréquents désignent les réalités les plus saillantes » (A. K o s e la k, 2003) et que « le secret de l'extraction du sens des mots réside dans leur cooccurrence » (J.-L. D e s s a 11 e s, 2019 : 59).

Tout au long du $\mathrm{XX}^{\mathrm{e}}$ siècle, les collocations ont soulevé l'intérêt de nombreux chercheurs comme O. Jespersen (1922), H.E. Palmer (1933), A. Renouf et J. Sinclair (1991) (cités par D. L e ga 11 o is, 2013). L'une des définitions les plus citées est celle de J.R. F i r th qui définit la collocation comme "statements of the habitual or customary places of that word" (1962 [1957]). Pour identifier les collocations dans un corpus, il faut s'appuyer sur une ou plusieurs mesures d'attraction entre les mots (ang. attraction between words) (S. Ever t, 2007 ; G. B o u m a, 2009). Le choix des mesures dépend de nombreux facteurs et peut mener à des 
résultats contradictoires. Il se complique davantage quand on veut comparer les résultats d'analyses de plusieurs corpus puisque différents corpus peuvent disposer de différentes mesures.

En premier lieu, il convient de mentionner la mesure la plus simple - la fréquence (Ch. Manning, H. S c hütze, 1999). On y part du principe que "If two words occur together a lot, then that is evidence that they have a special function that is not simply explained as the function that results from their combination" (1999: 143). L'inconvénient du critère de fréquence est qu'il considère les mots grammaticaux comme les plus fréquents. Pour y remédier, on emploie un filtre de parties du discours. L'autre problème est que "If the two constituent words of a frequent bigram like new companies are frequently occurring words (as new and companies are), then we expect the two words to co-occur a lot just by chance, even if they do not form a collocation" (1999: 162). De l'autre côté, une suite de mots peut être peu fréquente, tout en formant une forte collocation. Dans ces cas, le recours à d'autres méthodes plus sophistiquées de recherche de collocations permet de révéler des données linguistiques plus fines. Les mesures de collocations comme $\mathrm{MI}, \mathrm{Chi}^{\wedge} 2$ ou log-likelihood sont très répandues comme celles qui mesurent efficacement la force d'attraction entre les mots.

De plus, les mesures comme $\mathrm{Chi}^{\wedge} 2$ ou log-likelihood servent à prédire le voisinage d'un mot (S. Ever t, 2007). Cependant, quand on cherche à reconstruire la RLR, ce n'est pas la prédiction du mot voisin qui est essentielle, mais le sens qui est transmis par une combinaison de mots. Ainsi, souvent, les adjectifs très fréquents comme grand ne forment pas de collocations fortes (dont les mesures, p. ex. Chi^2 ou log-likelihood seraient très hautes), vu leur propre fréquence très élevée. À savoir, il serait difficile de prévoir un collocatif concret comme politesse d'un mot très fréquent comme grand puisqu'il a de nombreux collocatifs. Nonobstant, des expressions comme grande politesse $e^{1}$ sont importantes pour la compréhension du phénomène de politesse. Nous avons donc choisi de recourir à différentes mesures collocationnelles tout en gardant la distinction entre le terme cooccurrence, désignant les combinaisons lexicales fondées sur le critère de fréquence, et le terme de collocation, pour nommer les combinaisons lexicales fondées sur d'autres mesures de collocations.

${ }^{1}$ Grande politesse est la deuxième la plus fréquente cooccurrence parmi les cooccurrences adjectivales du mot politesse dans Frantext, dont la MI est pourtant basse $(4,87)$, beaucoup plus basse que la plus haute MI $(10,69)$ pour la collocation politesse obséquieuse. 


\section{Phénomène de politesse face à des problèmes terminologiques}

La politesse en tant que phénomène social et culturel est désignée par plusieurs mots aussi bien en français qu'en polonais. En français, le mot politesse étant le plus fréquent parmi tous ses synonymes ${ }^{2}$ d'après Frantext (fig. 2), nous avons décidé de l'analyser.



Fig. 2. Synonymes de politesse (Frantext)

En polonais, nous avons analysé quelques synonymes cités dans un dictionnaire des synonymes parmi lesquels le mot le plus fréquent est uprzejmość (2 fois plus fréquent que grzeczność d'après le NKJP, cf. fig. 3).

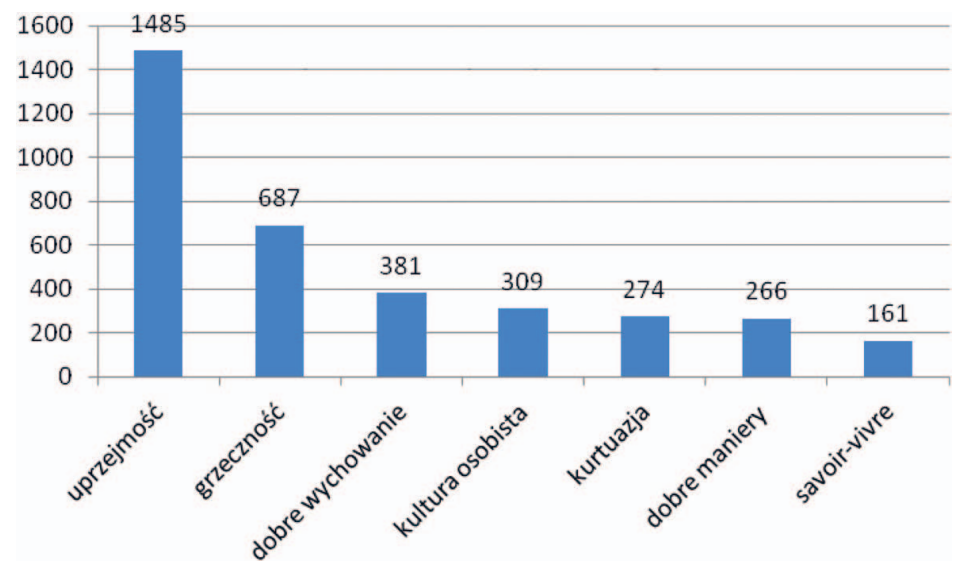

Fig. 3. Synonymes de grzeczność (NKJP)

${ }^{2}$ Surtout le mot courtoisie mérite que l'on s'y attarde dans prochaines analyses pour la RLR de politesse vu qu'il a un sens significativement différent par rapport au mot politesse ( $\mathrm{P}$. C h a r a u d e a u, 2014). 
Nous ne pouvons pourtant pas nous contenter de prendre en considération pour notre recherche uniquement ce mot vu le contexte linguistique où l'on parle des théories de grzeczność et non de uprzejmość. En définitive, nous avons décidé d'analyser les deux mots : uprzejmość et grzeczność.

La première conclusion qui en découle concerne la différence de fréquences et de fréquences par million de mots (fpm).

Tableau 1

Fréquences relatives et absolues (Frantext, NKJP)

\begin{tabular}{|c|c|c|}
\hline mot & fréquence & fpm \\
\hline politesse & 5921 & 23,40 \\
\hline uprzejmość & 1486 & 6,18 \\
\hline grzeczność & 687 & 2,86 \\
\hline
\end{tabular}

D'après l'analyse du NKJP et de Frantext, la fréquence absolue et la fpm de politesse sont significativement plus grandes que celles de uprzejmość et de grzeczność.

\section{L'étymologie et la définition des mots : politesse, grzeczność et uprzejmość}

Avant de passer à l'analyse de corpus sensu stricto, nous nous penchons sur deux éléments nécessaires de la reconstruction de la RLR, à savoir l'étymologie et les définitions dictionnairiques des trois mots analysés.

Le mot politesse vient de l'italien polito (fr. lisse, poli, propre). À partir de 1678 , le mot désigne " respect des règles de la bienséance ; bonne éducation » (TLFi).

Grzeczność est un mot polonais qui désignait un comportement juste et à propos (en ancien polonais $k$ rzeczy). Uprzejmość vient du tchèque et signifiait upřiemý / upřiemný (pl. prosty, fr. droit), szczery (fr. sincère), otwarty (fr. ouvert) (A. B ü ck n e r, 2000 [1927]). Tous les trois mots datent du XVI ${ }^{\mathrm{e}}$ siècle.

Le Grand Robert définit la politesse comme un « ensemble des règles, des usages qui régissent le comportement, le langage à adopter en société ».

Dans Słownik języka polskiego, nous pouvons lire que grzeczność est « une manière de comportement témoignant d'une bonne éducation » (pl. „sposób zachowania świadczący o dobrym wychowaniu", J. D o r o s ze w s k i, on-line, trad. - A.D.). Le même dictionnaire ne présente pas directement le mot uprzejmość, mais il renvoie à la définition de l'adjectif uprzejmy comme « se comportant conformément aux règles sociales, gentil envers les autres »(pl. „postępujący 
zgodnie z przyjętymi formami towarzyskimi, życzliwy wobec innych", J. Do r o s z e w s ki, on-line, trad. - A.D.).

Selon K. O żóg (1990), grzeczność est un système de normes socialement et universellement acceptées régissant un comportement dit poli, le comportement verbal inclus, des membres d'une communauté.

D'après ces définitions, il parait que la politesse est plus proche de grzeczność puisque, dans leurs définitions, l'on se réfère à une bonne éducation et au comportement verbal. Cependant, dans la définition de uprzejmość nous retrouvons le mot życzliwość (fr. gentillesse), qui élargit le sens du mot, en l'éloignant d'une simple conformité aux règles.

\section{Analyse de corpus - Corpora.uni-leipzig}

La première analyse de corpus est fondée sur les corpus accessibles sur le site Corpora.uni-leipzig (LC). L'avantage de ces corpus est l'homogénéité de la méthodologie adoptée, ce qui rend les résultats comparables. L'inconvénient, qui fait que l'on ne peut utiliser ce site qu'à titre indicatif, est que les outils de recherche dans le corpus polonais ne prennent pas en considération la déclinaison. De plus, le corpus français est beaucoup plus grand que le corpus polonais (respectivement 1468766604 mots vs 96476260 mots).

Dans le cadre de cette analyse, nous présentons d'abord les fréquences des trois mots politesse, uprzejmość et grzeczność dans les corpus : LC, Frantext, et NKJP et puis leurs cooccurrences recensées dans les corpus LC.

Tableau 2

Fréquences (LC, Frantext, NKJP)

\begin{tabular}{|l|c|c|c|c|}
\hline \multicolumn{1}{|c|}{ Mot } & $\begin{array}{c}\text { Fréquence } \\
\text { absolue (LC) }\end{array}$ & $\begin{array}{c}\text { Fréquence par million } \\
\text { de mots (fpm) (LC) }\end{array}$ & $\begin{array}{c}\text { Classe de } \\
\text { fréquence* (LC) }\end{array}$ & fpm \\
\hline $\begin{array}{l}\text { politesse (au singulier et } \\
\text { au pluriel) }\end{array}$ & 4061 & 2,76 & 14 & $\begin{array}{c}23,40 \\
\text { (Frantext) }\end{array}$ \\
\hline $\begin{array}{l}\text { uprzejmość } \\
\text { (tous les cas compris, au } \\
\text { singulier et au pluriel) }\end{array}$ & 373 & 3,87 & 13 & 6,18 (NKJP) \\
\hline $\begin{array}{l}\text { grzeczność (tous les cas } \\
\text { compris, au singulier et } \\
\text { au pluriel) }\end{array}$ & 134 & 1,39 & 14 & 2,86 (NKJP) \\
\hline
\end{tabular}

* Surtout la classe de fréquence est considérée comme un paramètre important puisqu'elle varie rarement parmi les corpus (https://wortschatz.uni-leipzig.de/en/documentation/faq\#corporaportal_wordinformation). Elle est calculée par rapport à la fréquence du mot le plus fréquent dans le corpus. Dans le corpus polonais en question, le mot polonais le plus fréquent est $w$, dans le corpus français, le mot le plus fréquent est de. 
En analysant les résultats des corpus LC, on constate que uprzejmość est un mot relativement plus fréquent que politesse et grzeczność puisque sa classe de fréquence est plus basse (13) que celle de politesse et grzeczność (14) et sa fréquence par million de mots est plus grande que celle des deux mots politesse et grzeczność. Pourtant, le mot politesse, tout en ayant la même classe de fréquence que grzeczność, est relativement plus fréquent que celui-là. Si l'on compare les fpm des trois mots analysés dans les corpus LC, Frantext et NKJF les résultats ne sont pourtant pas convergents. C'est le mot politesse qui est caractérisé (Frantext) par la fpm la plus grande $(23,40)$ que celle des uprzejmość et grzeczność (NKJP), cependant LC indique que la plus haute fréquence est celle du mot uprzejmość $(3,87)$.

Les graphiques ci-dessous (fig. 4-6) présentent des exemples des cooccurrences des trois mots analysés : politesse, uprzejmość et grzeczność dans LC.

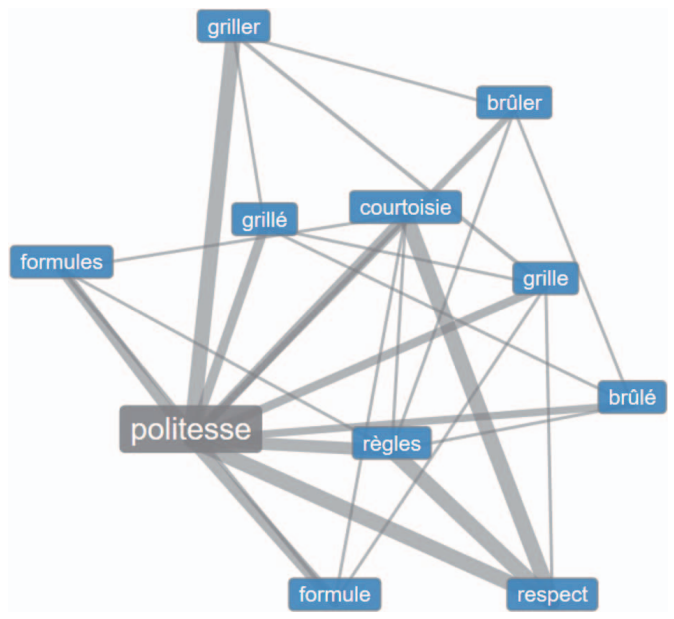

Fig. 4. Les cooccurrences du mot politesse (au singulier)

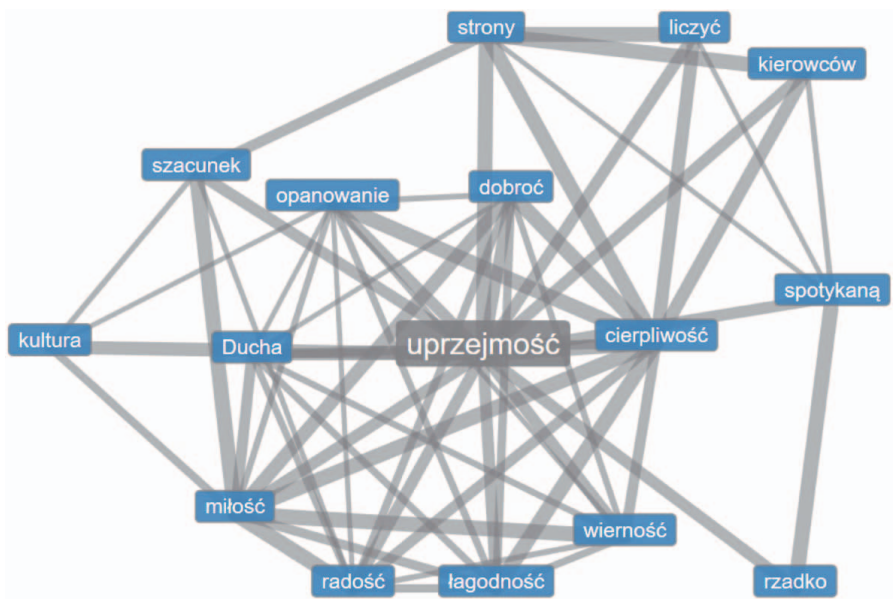

Fig. 5. Les cooccurrences du mot uprzejmość (au nominatif singulier) 


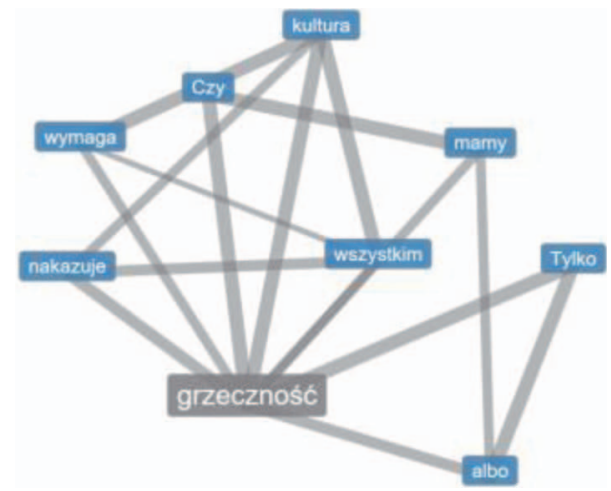

Fig. 6. Les cooccurrences du mot grzeczność (au nominatif singulier)

Dans le tableau 3, nous avons classé les cooccurrences présentées par les figures 4, 5, 6 en fonction des catégories suivantes : cooccurrences nominales, verbales, adjectivo-participiales et coordonnées.

Tableau 3

Tableau récapitulatif $(\mathrm{LC})$

\begin{tabular}{|l|l|l|l|l|}
\hline Cooccurrences & nominales & \multicolumn{1}{|c|}{ coordonnées } & \multicolumn{1}{c|}{ verbales } & \multicolumn{1}{c|}{$\begin{array}{c}\text { adjectivo- } \\
\text { participiales }\end{array}$} \\
\hline politesse & $\begin{array}{l}\text { formules, } \\
\text { règles }\end{array}$ & respect, courtoisie & brûler, griller & brûlée, grillée \\
\hline uprzejmość & $\begin{array}{l}\text { kierowców } \\
\text { (de chauf- } \\
\text { feurs) }\end{array}$ & $\begin{array}{l}\text { cierpliwość (patience) } \\
\text { łagodność (douceur), } \\
\text { dobroć (bonté), opanowanie } \\
\text { (maîtrise de soi), kultura, } \\
\text { wierność (fidélité), szacunek } \\
\text { (respect), radość (joie), } \\
\text { miłość (amour) }\end{array}$ & liczyć (compter) & $\begin{array}{l}\text { (rzadko) spoty- } \\
\text { kana (rarement } \\
\text { rencontrée) }\end{array}$ \\
\hline grzeczność & kultura & $\begin{array}{l}\text { nakazuje } \\
\text { (impose), } \\
\text { wymaga (exige, } \\
\text { oblige) }\end{array}$ & \\
\hline
\end{tabular}

En analysant ce tableau, nous pouvons constater peu de ressemblances parmi les cooccurrences. Les cooccurrences adjectivo-participiales sont peu nombreuses, ce qui parait peu intuitif : juste deux exemples pour uprzejmość, deux pour politesse, aucun pour grzeczność. Les cooccurrences coordonnées sont les plus nombreuses, surtout pour le mot uprzejmość. Par conséquent, nous avons décidé d'approfondir notre analyse de deux types de cooccurrences, adjectivo-participiales et coordonnées en nous référant aux corpus Frantext et NKJP (équilibré). 


\section{Analyse de corpus - Frantext et NKJP}

Dans cette partie, nous analysons les collocations des trois mots politesse, uprzejmość et grzeczność avec les adjectifs ou les participes passés ${ }^{3}$ (les modèles de parties du discours AN (adjectif + nom), NA (nom + adjectif), PPN (participe passé + nom), NPP ( + participe passé) dont la fréquence dans Frantext et NKJP est plus grande ou égale à $5^{4}$. Les collocations que nous avons recueillies sont représentées par les graphiques (fig. 7-9) :

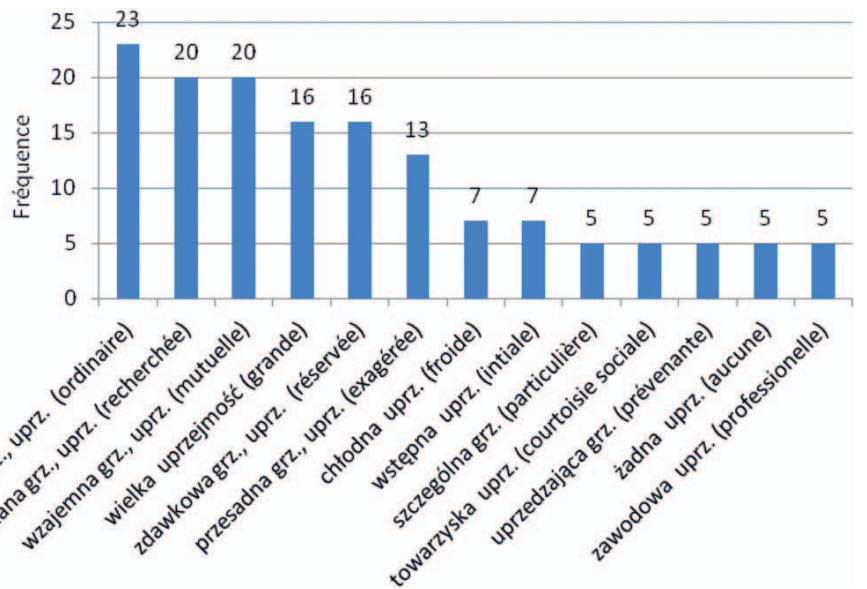

Fig. 7. Collocations A-PPN, NA-PP de grzecznossć et/ou uprzejmość (NKJP, f >=5)

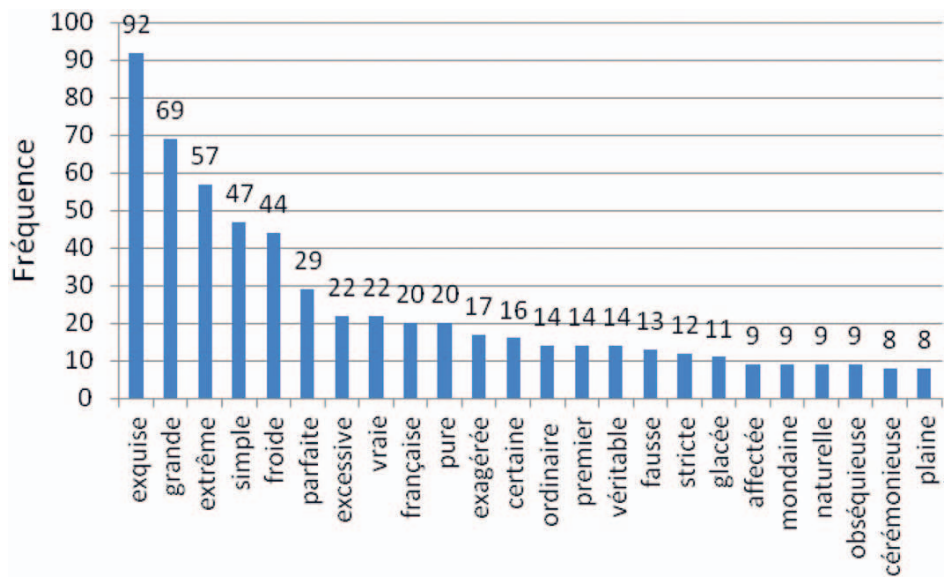

Fig. 8. Collocations AN, NA, PPN, NPP de politesse (Frantext, $\mathrm{f}>=8$ )

\footnotetext{
${ }^{3}$ Dans Frantext, les collocatifs analysés ont été annotés comme : adjectifs ou verbe participe passé.

${ }^{4}$ Un seuil indiqué par S. Evert (2007).
} 


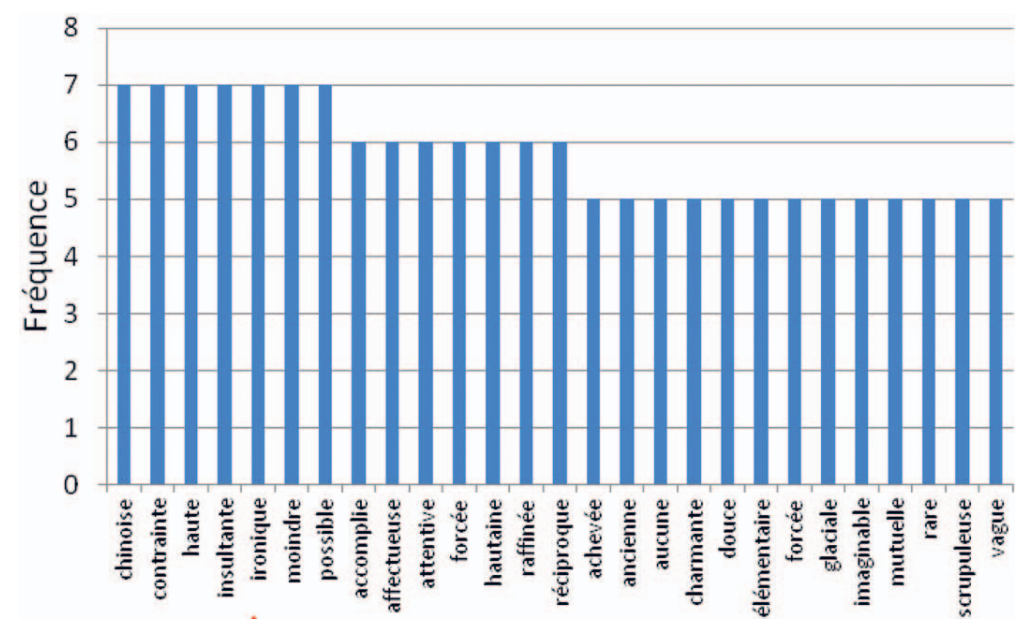

Fig. 9. Collocations AN, NA, PPN, NPP de politesse (Frantext, $8>\mathrm{f}>=5$ )

Nous avons traduit les adjectifs polonais en français, pour approcher le sens des collocations polonaises. Ce ne sont que des traductions approximatives pour deux raisons : premièrement, elles sont hors contexte, et deuxièmement, à cause de difficultés terminologiques. Par exemple, en polonais l'on parle de chłodna uprzejmość que nous traduisons comme politesse froide, pourtant chłodna veut dire tiède ou frais.

Parmi les collocations analysées, il y a plus de collocations en français 50 contre 13 en polonais. Parmi ces collocations, nous pouvons indiquer 4 ressemblances : en polonais et en français, l'on parle de politesse ordinaire, exagérée, froide, grande. En polonais, l'on parle de wyszukana grzeczność ou wyszukana uprzejmość, qui sont très fréquentes, que nous traduisons comme politesse recherchée. L'adjectif recherché, à notre sens, est proche de exquis, mais n'a pas exactement le même sens et surtout pas la même connotation, ce que nous allons montrer plus tard.

En vue d'une analyse plus fine, nous avons classé toutes les collocations (dont la fréquence est plus grande ou égale à 5 en trois catégories : catégorie à sens positif, à sens négatif, à sens neutre, selon la conception de prosodie sémantique (B. Louw, 1993 cité par R. Lo ock, 2016 ; H. Ty ne, 2019). 
Politesse - prosodie sémantique

\begin{tabular}{|c|c|c|}
\hline \multicolumn{3}{|c|}{ Connotation positive, négative ou neutre de collocations de politesse } \\
\hline positive & négative & neutre \\
\hline 1. exquise & 1. froide & 1. simple \\
\hline 2. grande & 2. exagérée & 2. ordinaire \\
\hline 3. extrême & 3. fausse & 3. française \\
\hline 4. parfaite & 4. glacée & 4. stricte \\
\hline 5. pure & 5. obséquieuse & 5. naturelle \\
\hline 6. vraie & 6. ironique & 6. mondaine \\
\hline 7. véritable & 7. excessive* & 7. mutuelle \\
\hline 8. affectée & 8. obséquieuse & 8. possible \\
\hline 9. raffinée & 9. vague & 9. première \\
\hline 10. haute & 10. rare* & 10. élémentaire \\
\hline 11. accomplie & 11. glaciale & 11. réciproque \\
\hline 12. excessive* & 12. forcée & 12. chinoise \\
\hline 13. scrupuleuse & 13. achevée* & \\
\hline 14. imaginable & 14. hautaine & \\
\hline 15. douce & 15. aucune & \\
\hline 16. charmante & & \\
\hline 17. ancienne & & \\
\hline 18. achevée* & & \\
\hline 19. attentive & & \\
\hline 20. affectueuse & & \\
\hline 21. rare* & & \\
\hline
\end{tabular}

Tableau 5

Grzeczność et/ou uprzejmość — prosodie sémantique

\begin{tabular}{|l|l|l|}
\hline \multicolumn{2}{|c|}{ Connotation positive, négative ou neutre de collocations de grzeczność et/ou uprzejmość } \\
\hline \multicolumn{1}{|c|}{ positive } & \multicolumn{1}{|c|}{ négative } & \multicolumn{1}{c|}{ neutre } \\
\hline wyszukana (recherchée)* & wyszukana* & zwykła (ordinaire) \\
wielka (grande) & zdawkowa (réservée) & wzajemna (mutuelle) \\
szczególna (particulière) & przesadna (exagérée)* & wstępna (initiale) \\
uprzedzająca (prévenante)* & chłodna (froide) & towarzyska (courtoisie) \\
& żadna (aucune) & zawodowa (professionnelle) \\
\hline
\end{tabular}

Tous les termes marqués par un astérisque sont ambigus puisque leur connotation varie en fonction du contexte. En effet, les collocations comme uprzejmość / grzeczność wyszukana (fr. recherchée) ou prévenante peuvent être positives, mais elles ont souvent une connotation négative quand elles désignent des comportements exagérés pour dissimuler une intention peu sincère, voire malveillante ${ }^{5}$. Il convient pourtant de souligner qu'en France aussi « l'excessive courtoisie

${ }^{5}$ Nous retrouvons ce sens dans un passage du roman La poupée de B. Prus : „Maruszewicz zobaczywszy wstrętną figurę kupczyka, doświadczył tak rozmaitych uczuć, że nie tylko nie wiedział, co mówi, ale nawet o czym myśli. Pamiętał tylko, że Wokulski zaprowadził go do gabinetu za sklepem, gdzie znajdowała się żelazna kasa, i powiedział sobie, że uczucia, jakich doznaje na widok 
a quelque chose de suspect, d'hypocrite » (P. C h a r a u d e a u, 2014), mais le mot qui nécessite une explication est le mot excessif, désignant des réalités différentes en polonais et en français. À notre sens, un comportement perçu comme une politesse excessive, voire suspecte en polonais serait souvent acceptable pour les Français et caractérisé par les adjectifs exquis, grand ou extrême.

La composante positive en polonais est moins importante que les composantes négatives et neutres vu qu'elle ne contient que deux adjectifs : grand et particulier. En français cette composante contient 21 adjectifs et elle est plus importante que les composantes négatives et neutres. L'on peut en déduire que le mot politesse a une prosodie sémantique positive plus forte que ses deux équivalents français.

\subsection{Collocations adjectivales et participiales}

Étant donné que le critère de fréquence n'est pas un bon indicateur d'une « force d'attraction » entre les mots et que les corpus analysés offrent des mesures statistiques de collocations différentes qui, en conséquence, ne permettent pas de comparer les résultats, nous avons cherché des collocations en calculant l'Information mutuelle (ang. Mutual Information, MI).

Tableau 6

MI pour la politesse dans Frantext

\begin{tabular}{|l|c|c|}
\hline \multicolumn{1}{|c|}{ Adjectif ou participe passé } & $\begin{array}{c}\text { Fréquence dans Frantext } \\
0=\mathrm{f}(\mathrm{adj}, \mathrm{pp} \mid \text { politesse })\end{array}$ & MI \\
\hline obséquieux & 9 & 10,69 \\
\hline exquis & 92 & 9,52 \\
\hline cérémonieux & 8 & 9,50 \\
\hline insultant & 7 & 9,20 \\
\hline stricte & 12 & 8,48 \\
\hline exagéré & 17 & 8,12 \\
\hline pur & 20 & 7,79 \\
\hline imaginable & 5 & 7,72 \\
\hline excessif & 22 & 7,40 \\
\hline raffiné & 6 & 7,34 \\
\hline
\end{tabular}

Wokulskiego, są lekceważeniem pomieszanym ze wzgardą. Później przypomniał sobie, że afekta te starał się zamaskować wyszukaną grzecznością, która nawet w jego oczach wyglądała na pokorę" (Lalka, B. Prus d'après NKJP). (" Maruszewicz, voyant la figure dégoûtante du marchand, éprouvait une telle variété de sentiments qu'il ne savait pas seulement ce qu'il disait, mais même ce qu'il pensait. Il se souvenait seulement que Wokulski l'avait conduit au bureau derrière la boutique, où se trouvait une caisse de fer, et se dit que les sentiments qu'il éprouvait à l'égard de Wokulski étaient du dédain mêlé de mépris. Il se rappela plus tard qu'il essayait de masquer ces émotions par une politesse recherchée, qui même à ses yeux avait l'air humble » [trad. — AD]). 
Tableau 7

MI de grzeczność dans NKJP

\begin{tabular}{|l|c|c|}
\hline \multicolumn{1}{|c|}{ Adjectif droite + gauche } & $\begin{array}{c}\text { Fréquence dans NKJP : } \\
0=\mathrm{f}(\operatorname{adj} \mid \text { grzeczność) }\end{array}$ & MI \\
\hline uprzedzająca (prévenante) & 5 & 14,55 \\
\hline wyszukana (recherchée) & 13 & 10,49 \\
\hline przesadna (exagérée) & 5 & 11,78 \\
\hline zdawkowa (réservée) & 9 & 8,17 \\
\hline wzajemna (mutuelle) & 9 & 7,03 \\
\hline zwykła (ordinaire) & 5 & 5,87 \\
\hline szczególna (particulière) & & \\
\hline
\end{tabular}

MI de uprzejmość dans NKJP

Tableau 8

\begin{tabular}{|l|c|c|}
\hline \multicolumn{1}{|c|}{ Adjectif (droite, gauche) } & $\begin{array}{c}\text { Fréquence dans NKJP : } \\
0=\mathrm{f}(\mathrm{adj} \mid \text { grzeczność) }\end{array}$ & MI \\
\hline wyszukana (recherchée) & 7 & 10,04 \\
\hline zdawkowa (réservée) & 11 & 11,81 \\
\hline przesadna (exagérée) & 8 & 10,06 \\
\hline chłodna (froide) & 7 & 7,49 \\
\hline zwykła (ordinaire, simple) & 14 & 6,55 \\
\hline wstępna (initiale) & 7 & 6,71 \\
\hline wzajemna (mutuelle) & 11 & 7,35 \\
\hline towarzyska (courtoisie sociale) & 5 & 6,74 \\
\hline wielka (grande) & 16 & 4,12 \\
\hline zawodowa (professionnelle) & 5 & 4,19 \\
\hline żadna (aucune) & 5 & 2,66 \\
\hline
\end{tabular}

Parmi les trois collocations les plus fortes du mot politesse, il y en a une qui a une connotation positive - politesse exquise. Par contre, parmi les trois collocations les plus fortes des mots uprzejmość et grzeczność, il n'y a que des collocations à connotation négative excepté la collocation avec l'adjectif recherché qui peut être positive ou négative. L'analyse des collocations les plus fortes fondées sur la MI n'indique pas de grandes différences par rapport aux résultats cités avant pour la fréquence. 


\subsection{Cooccurrences coordonnées}

Ce type de cooccurrences est conforme au modèle de parties du discours NCN (nom-conjonction-nom) et NN (nom-(virgule)-nom). Quelques exemples de ce type de collocations tirés de Frantext et de NKJP sont présentés dans les tableaux $9-11$.

Tableau 9

Cooccurrences coordonnées de politesse

\begin{tabular}{|c|c|}
\hline $\begin{array}{l}\text { Cooccurrences de politesse } \\
\text { à caractère : }\end{array}$ & Exemples - fr \\
\hline éthique & «[...] qui les reçurent avec politesse et bonté » \\
\hline esthétique & $\begin{array}{l}\text { "Chacun apportait à ces petites réunions sa politesse exquise } \\
\text { et ses belles manières. " }\end{array}$ \\
\hline intellectuel & $\begin{array}{l}\text { «[...] mais partageons aussi la loyauté, la politesse et } \\
\text { l'esprit ! » }\end{array}$ \\
\hline sentiment & $\begin{array}{l}\text { «[...] avec un compliment tel que la politesse et l'amour } \\
\text { peuvent le dicter. » }\end{array}$ \\
\hline attitude / comportement & « Douceur et politesse exquise des habitants. » \\
\hline autres & « on y trouve aujourd'hui $[\ldots]$ la gloire et la politesse » \\
\hline
\end{tabular}

Tableau 10

Cooccurences coordonnées de grzeczność

\begin{tabular}{|l|l|}
\hline \multicolumn{1}{|c|}{$\begin{array}{c}\text { Cooccurrences de grzeczność } \\
\text { à caractère : }\end{array}$} & \multicolumn{1}{c|}{ Exemple - pl } \\
\hline éthique & $\begin{array}{l}\text { „,to ich uczyło pokory i grzeczności” } \\
\text { „a także jej grzeczność i dobre serce” } \\
\text { „Zaleca się im grzeczność i życzliwość” }\end{array}$ \\
\hline esthétique & $\begin{array}{l}\text {,To jednak sprawa dobrego wychowania i grzeczności, } \\
\text { a nie zasada mądrości, co wynika z wysokiej kultury } \\
\text { osobistej, elegancji i grzeczności” }\end{array}$ \\
\hline intellectuel & „rozsądek i grzeczność nakazywał” \\
\hline émotionnel & „niepomny grzeczności i przyjaźni dla swego kamrata” \\
\hline attitude / comportement & $\begin{array}{l}\text { „Wymieniamy uśmiechy i grzeczności” } \\
\text { „Byliśmy jedynie obserwatorami, zaproszonymi z grzeczności } \\
\text { i szacunku” }\end{array}$ \\
\hline autres & „Uczyliśmy ich grzeczności i uczciwej pracy” \\
\hline
\end{tabular}


Cooccurrences coordonnées de uprzejmość

\begin{tabular}{|c|c|}
\hline $\begin{array}{c}\text { Cooccurrences de uprzejmość } \\
\text { à caractère : }\end{array}$ & Exemples - pl \\
\hline éthique & $\begin{array}{l}\text { „był wzorem dobroci i uprzejmości. Jego skromność } \\
\text { i uprzejmość” }\end{array}$ \\
\hline esthétique & $\begin{array}{l}\text { „nie wyniósł najlepszego zdania o manierach } \\
\text { i uprzejmości Granta." }\end{array}$ \\
\hline intellectuelle & „dzięki bystrości i uprzejmości pana Merthona” \\
\hline sentiment & $\begin{array}{l}\text { "Mówi [...] o sympatii i uprzejmości, z którą spotkał się } \\
\text { w Londynie" }\end{array}$ \\
\hline attitude / comportement & $\begin{array}{l}\text { „traktowali się nawzajem z całym możliwym szacunkiem } \\
\text { i uprzejmością" }\end{array}$ \\
\hline autres & „dzięki kompetencji i uprzejmości policjantów” \\
\hline
\end{tabular}

Toutes les cooccurrences coordonnées ont été classées en 6 catégories : éthique, esthétique, intellectuel, émotionnel, attitude/comportement, et autres. Ce n'est pas le sens de toute la cooccurrence qui est pris en considération dans le classement, mais le sens du collocatif. Ainsi, dobroć i uprzejmość (fr. bonté et politesse) est classé dans la catégorie " éthique » puisque le mot bonté a une connotation éthique, szacunek i uprzejmość (fr. respect et politesse) est classé dans la catégorie « attitude et comportement » à cause de la connotation du mot respect.

Après avoir catégorisé toutes les collocations coordonnées, nous avons obtenu les résultats qui sont présentés dans les graphiques (fig. 10-12).

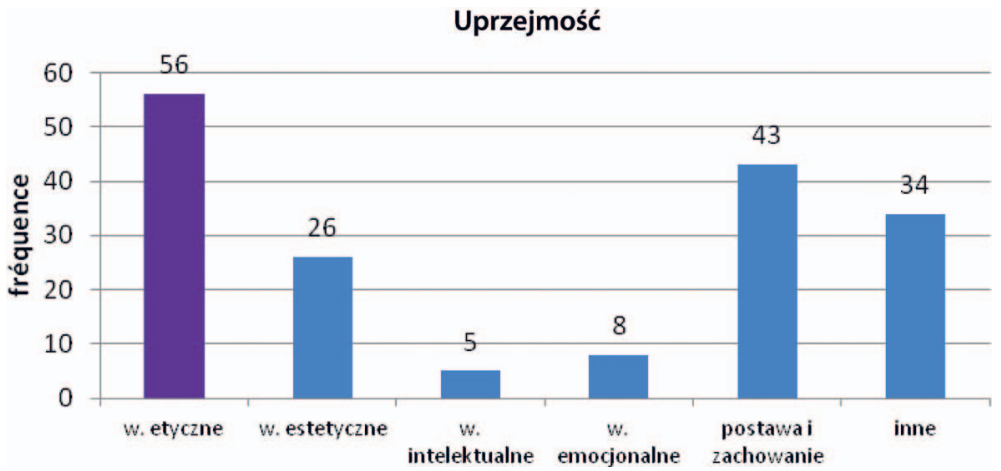

Fig. 10. Différentes composantes de uprzejmość 


\section{Grzeczność}

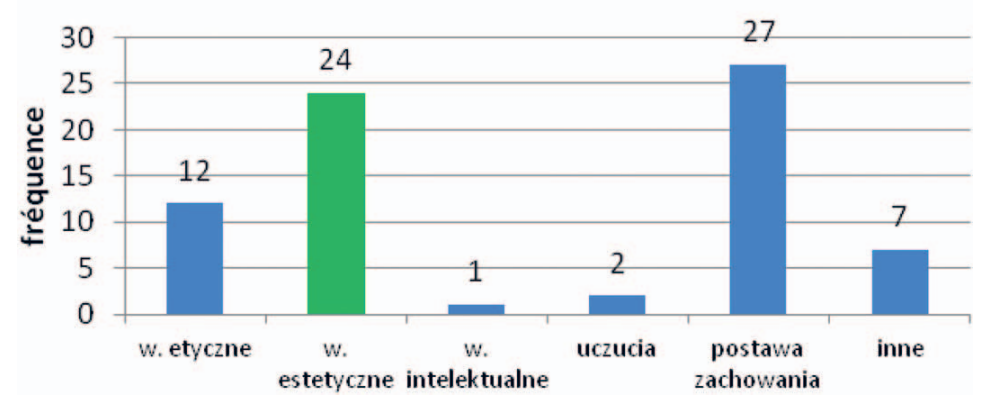

Fig. 11. Différentes composantes de grzeczność

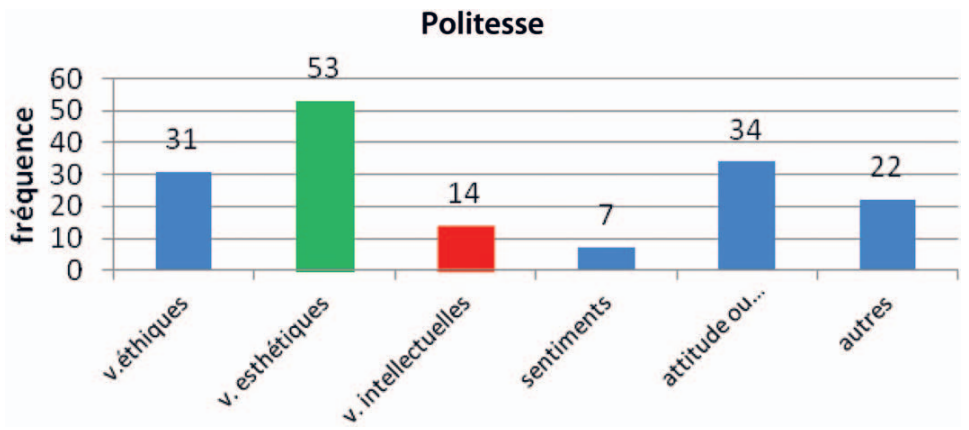

Fig. 12. Différentes composantes de politesse

Les fréquences qui sont présentées dans les graphiques ci-dessus sont des fréquences sommatives absolues de toutes les fréquences absolues de tous les éléments constitutifs d'une catégorie. En comparant les trois graphiques, nous observons d'abord que la distribution de toutes les catégories est différente pour chaque mot. La première différence concerne la catégorie dominante pour chaque mot. La catégorie dominante de uprzejmość est la catégorie " éthique », qui apparait dans le NKJF avec la fréquence 56 avec les noms les plus fréquents : $\dot{y y c z l i w o s ́ c ́}$ (fr. gentillesse, 14 dans NKJP), dobroć (fr. bonté, 5 fois), skromność (fr. modestie, 4 fois), cierpliwość (fr. patience, 3 fois), uczciwość (fr. honnêteté, 3 fois). Ce n'est pas le cas de politesse dont la catégorie dominante est la catégorie « esthétique » qui apparait avec la fréquence 53 avec les substantifs les plus fréquents : grâces ( 8 fois dans Frantext), manières (4 fois), formes ( 3 fois). Cette catégorie est aussi importante (la deuxième) pour le mot grzeczność avec les noms les plus fréquents : uktadność (fr. galanterie, courtoisie, 5 fois dans NKJP), dobre maniery (fr. bonnes manières, 3 fois), elegancja (fr. élégance, 2 fois). En général, on observe une certaine ressemblance entre les distributions des catégories analysées des mots grzeczność et politesse. 
Une autre ressemblance est la position de la catégorie « attitude et comportement » dans la distribution de toutes les catégories des trois mots analysés. C'est une catégorie très importante pour tous les mots. Elle est dominante pour le mot grzeczność (fréquence 27) avec les collocatifs les plus fréquents : szacunek, uszanowanie (fr. respect, 7 fois dans NKJP), spokój (fr. calme, 3 fois) et la deuxième catégorie la plus fréquente pour le mot uprzejmość (la fréquence 47) avec les collocatifs : uśmiech (fr. sourire, 12 fois dans NKJP), szacunek (fr. respect, 7 fois) et pour le mot politesse (fréquence 34 ) avec les collocatifs les plus fréquents : douceur (4 fois dans Frantext), savoir-vivre (3 fois), délicatesse (2 fois), sourire ( 2 fois), calme ( 2 fois). Il convient d'indiquer la présence du mot respect pour les deux mots polonais et du mot gentillesse dans la catégorie " éthique " du mot uprzejmość. La « haute position » de respect et de gentillesse comme collocatifs coordonnés de grzeczność et uprzejmość confirme les observations de K. Ożóg $(1990,2001)$ concernant les principes fondamentaux de la politesse dans la perspective polonaise.

Dans la figure 12, nous observons la catégorie " intellectuelle », qui n'est pas dominante pour le mot politesse, mais qui est plus significative que pour les mots polonais. Dans cette catégorie, il y a des substantifs comme esprit (fréquence - 8), prudence (fréquence - 3), raison (une fois), curiosité (une fois), goût (une fois). C'est surtout le mot esprit qui nous parait important puisque la collocation esprit et politesse parait 8 fois dans le corpus. À notre sens, c'est probablement le caractère « intellectuel » de la politesse française, notamment l'« esprit français » qui a forgé la politesse française dont témoignent les cooccurrences comme politesse exquise, raffinée, mondaine et justement française. Il est aussi à noter que le mot esprit est aussi un mot chargé culturellement qui, au demeurant, n'a pas son équivalent en polonais, ce qui ouvre de nouvelles perspectives de recherche.

Le graphique suivant (fig. 13) représente la distribution des 6 catégories du mot politesse et les composantes sommatives des deux mots polonais. La catégorie dominante de la politesse est la catégorie « esthétique », par contre la politesse polonaise désignée par les mots grzeczność et uprzejmość est surtout caractérisée par les catégories « éthique » et « attitude et comportement». 


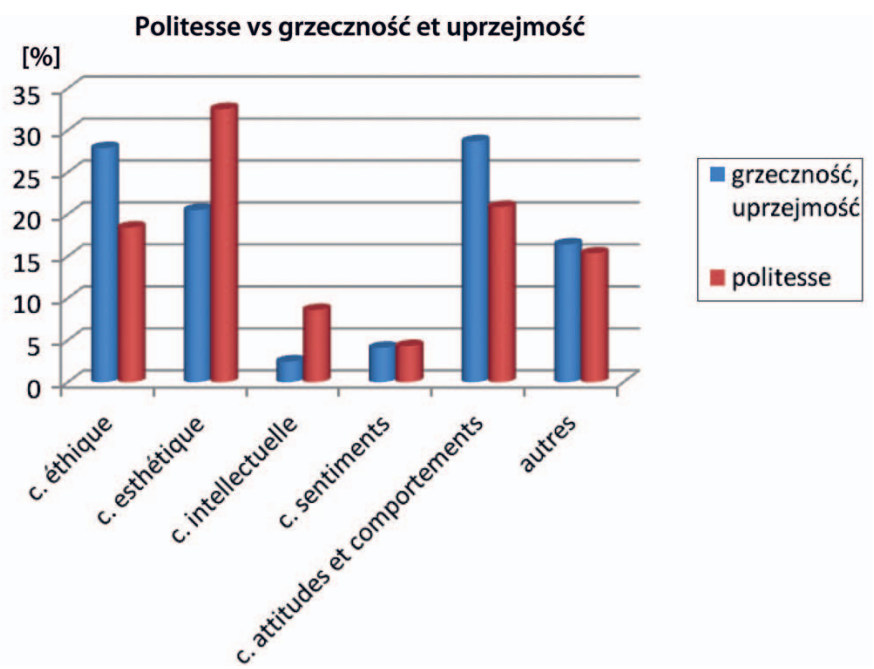

Fig. 13. Composantes sommatives

\subsection{Ressemblance exprimée par une corrélation}

Le paramètre suivant qui peut fournir une information sur la ressemblance entre les trois mots : politesse, grzeczność et uprzejmość est le coefficient de corrélation linéaire (CCL) entre les distributions des six catégories des cooccurrences coordonnées analysées ci-dessus. Nous avons obtenu les résultats suivants :

\section{CCL grzeczność vs politesse $=\mathbf{0 . 8 6}$ \\ CCL uprzejmość vs politesse $=\mathbf{0 . 5 2}$ \\ CCL grzeczność vs uprzejmość $=\mathbf{0 . 5 7}$}

qui permettent de constater qu'il existe une plus grande similitude entre les mots grzeczność et politesse $(\mathrm{CCL}=0.86)$ qu'entre uprzejmość et politesse $(\mathrm{CCL}=$ 0.52), et paradoxalement, qu'entre les deux mots polonais grzeczność et uprzejmość $(\mathrm{CCL}=0.57)$, que l'on considère intuitivement comme de très proches synonymes.

\section{Conclusions}

Pour terminer, nous tenons à souligner quelques conclusions qui découlent de notre recherche. Premièrement, la fréquence par million de mots (fpm) est plus haute pour politesse (Frantext) que pour grzeczność et uprzejmość dans NKJP (ce qui n'est pourtant pas confirmé par le corpus LC, la fpm de uprzejmość étant 
la plus grande). Ensuite, il y a plus de cooccurrences adjectivo-participiales et coordonnées de politesse dans Franext que de grzeczność et uprzejmość dans le NKJP.

Deuxièmement, il est à noter que la prosodie sémantique fondée sur la composante positive des collocations adjectivo-participiales de politesse est significativement plus forte que celle des deux mots polonais.

Troisièmement, d'après l'analyse des cooccurrences coordonnées, la politesse a un caractère plus esthétique que la politesse polonaise, surtout celle désignée par le mot uprzejmość, qui est dominée plutôt par des aspects éthiques et ceux liés aux attitudes et aux comportements. Enfin, il y a une haute ressemblance (corrélation) entre politesse et grzeczność, ce qui a été suggéré par les analyses étymologique et dictionnairique.

Ces résultats fournissent une explication concernant le caractère des comportements polis des Polonais et des Français. Les Polonais, associant leur comportement poli surtout au respect, à la gentillesse et à la bonté, « limitent » leur politesse à un cercle de leurs proches ou de personnes qu'ils connaissent bien et respectent (tout en gardant un minimum de politesse envers les autres). Dans les contacts en dehors du cercle de proches, c'est une politesse, dirait-on, " négative », en recourant à la terminologie de G. Leech (cité par A. Żu rek, 2008), dont l'objectif est de minimiser des situations potentiellement conflictuelles. Pour les Français, la politesse, associée à des phénomènes de nature plutôt esthétique et intellectuelle, plus superficiels et moins engageant personnellement, permet un comportement poli dans un plus grand nombre de situations dans la vie sociale, largement dépassant le cercle de proches. Dans cette perspective, la politesse française s'inscrirait dans le type de "politesse positive » qui maximise le comportement poli (G. Le e ch, 1983 cité par A. Ż u re k, 2008).

Il est à noter que la reconstruction de la RLR de la politesse en français et en polonais fondée sur l'analyse de corpus exige encore deux précisions. En premier lieu, nos conclusions, reposant sur la validité des classements et des catégorisations effectués, pourront être plus fiables si cette validité est plus grande, ce qui sera possible grâce aux jugements de plusieurs classificateurs traités ensuite par une mesure statistique (kappa de Fleiss) permettant d'évaluer la concordance des assignations qualitatives faites par ces classificateurs.

En deuxième lieu, afin d'accomplir notre analyse, nous envisageons d'examiner les collocations nominales et verbales des mots politesse, grzeczność et uprzejmość, et ensuite d'analyser les collocations des adjectifs poli, grzeczny, uprzejmy. Ainsi disposerons-nous d'une méthodologie complète et de résultats fiables qui permettront de développer une approche lexicale inductive en didactique du FLE afin de faciliter à l'apprenant la conceptualisation des notions complexes désignées par des mots chargés culturellement. 


\section{Références citées}

B o u m a G., 2009 : "Normalized (Pointwise) Mutual Information in Collocation Extraction". Proceedings of GSCL [Department Linguistik, Universität Potsdam]. https:// pdfs.semanticscholar.org/1521/8d9c029cbb903ae7c729b2c644c24994c201.pdf (consulté le 27 décembre 2019).

B row n P., L e vi n s o n S., 1987 : Politeness: Some universals in language usage. Cambridge: Cambridge University Press. https://www.academia.edu/26395652/Politeness_Some_universals_in_language_usage (consulté le 11 juillet 2020).

B r ü ck ne r A., 2000 [1927] : Słownik etymologiczny języka polskiego. Warszawa, Wiedza Powszechna.

Charaude a u P., 2014 : «Étude de la politesse entre communication et culture ». In : A.M. Cozma, A. Bella ch hab, M. P e s che ux, éds. : Du sens à la signification. De la signification aux sens. Mélanges offerts à Olga Galatanu. Bruxelles, PIEPeter Lang, 137-154. http://www.patrick-charaudeau.com/Etude-de-la-politesseentre.html (consulté le 27 décembre 2019).

D e s s a 11 e s J.-L., 2019 : Des intelligences très artificielles. Paris, Odile Jacob.

D i V i to S., 2013 : «L'utilisation des corpus dans l'analyse linguistique et dans l'apprentissage du FLE ». Linx 68-69, 159-176. https://journals.openedition.org/linx/1519 (consulté le 27 décembre 2019).

Dryjańska A., 2016 : „Słowa-wartości: interkulturowość ukryta w językach”. Neofi$\operatorname{lolog} 46 / 2,37-51$.

Evert S., 2007 : Corpora and collocations. Osnabrück, Institute of Cognitive Science, University of Osnabrück. http://www.stefan-evert.de/PUB/Evert2007HSK_extended_ manuscript.pdf (consulté le 27 décembre 2019).

Firth J.R., 1962 [1957] : A Synopsis of Linguistic Theory. 1930-1955. Oxford, Basil Blackwell. https://annabellelukin.edublogs.org/files/2013/08/Firth-JR-1962-A-Synopsis-of-Linguistic-Theory-wfihi5.pdf (consulté le 11 novembre 2020).

Francesco A., 2010 : «De l'approche lexiculturelle des dictionnaires monolingues : le nouveau Petit Robert 2010, version électronique ». Études de linguistique appliquée 1, 157, 9-22. https://www.cairn.info/revue-ela-2010-1-page-9.htm (consulté le 27 décembre 2019).

Galis s on R., 1988 : «Cultures et lexicultures. Pour une approche dictionnairique de la culture partagée ». Cahiers d'Études Hispaniques Médiévales, 325-341. https:// www.persee.fr/doc/cehm_0180-9997_1988_sup_7_1_2133 (consulté le 11 juillet 2020).

Galis s on R., 2005 : «La pragmatique lexiculturelle pour accéder autrement, à une autre culture, par un autre lexique ». Mélanges CRAPEL 2, 47-73. http://www.atilf. fr/spip.php?article3471 (consulté le 27 décembre 2019).

Goffman E., 2005 [1967] : Interaction Ritual: Essays in Face to Face Behavior. New Brunswick, Transaction Publishers. https://books.google.pl/books?hl=fr\&lr=\&id=qD hd138pPBAC\&oi=fnd\&pg=PR7\&dq=goffman+interaction + rituals\&ots $=9$ j32KZeJxp $\& \operatorname{sig}=$ pkkbr5iYnacc9JX7q6EM3f6GRTo\&redir_esc $=\mathrm{y} \# \mathrm{v}=$ onepage $\& \mathrm{q}=$ goffman $\% 20$ interaction $\% 20$ rituals\&f=false (consulté le 11 juillet 2020). 
Kerbrat-Orecchioni C., 2010 : « L'impolitesse en interaction ». Lexis - Journal in English Lexicology. https://journals.openedition.org/lexis/796 (consulté le 11 juillet 2020).

Koselak A., 2003 : «La sémantique naturelle d'Anna Wierzbicka et les enjeux interculturels ». Questions de communication 4, 83-95. https://doi.org/10.4000/questionsdecommunication.4611 (consulté le 11 juillet 2020).

Lak off R., Ide S., 2005 : Broadening the Horizon of Linguistic Politeness. Amsterdam/Philadelphia, John Benjamins.

L e e ch G., 1983 : Principles of Pragmatics. London, New York, Longman. https://books. google.pl/books?hl=fr\&lr=\&id=2uWXCwAAQBAJ\&oi=fnd\&pg=PP1\&dq=leech + pri nciples + of + pragmatics + pdf\&ots $=$ KAsVxfBtYh\&sig=W4DGC1MEQaAXDzNJEXN WyKZkMCE\&redir_esc=y\#v=onepage \&q\&f=false (consulté le 11 juillet 2020).

L e ga 11 o is D., 2013 : «La colligation : autre nom de la collocation grammaticale ou autre logique de la relation mutuelle entre syntaxe et sémantique ? ». Corpus 11. http://journals.openedition.org/corpus/2202 (consulté le 27 décembre 2019).

L o ock R., 2006 : La traductologie de corpus. Lille, Presses Universitaires du Septentrion. https://books.google.fr/books?id=90SqDQAAQBAJ\&pg=PA121\&lpg=PA1 $21 \& \mathrm{dq}=$ prosodie + semantique + Louw $+1993 \&$ source $=$ bl\&ots $=$ be 6 ObTdDOL $\&$ sig $=$ AC fU3U2bhzudRCwuqmhMk4ht3SC6A8Mtfw\&hl=fr\&sa=X\&ved=2ahUKEwi79df9v uTjAhWE6aQKHVqoDr4Q6AEwA3oECAkQAQ\#v=onepage\&q=prosodie\&f=false (consulté le 27 décembre 2019).

Manning Ch., Schütze H., 1999 : Foundations of statistical natural language processing. Cambridge, Massachusetts, London, England, The MIT Press. https://www. cs.vassar.edu/ cs366/docs/Manning_Schuetze_StatisticalNLP.pdf (consulté le 27 décembre 2019).

Marcjanik M., 1997 : Polska grzeczność językowa. Kielce, Wyższa Szkoła Pedagogiczna.

O żó g K., 1990 : Zwroty grzecznościowe współczesnej polszczyzny mówionej. Kraków, Warszawa, Państwowe Wydawnictwo Naukowe.

Ożóg K., 2001 : Polszczyzna przełomu XX i XXI. Wybrane zagadnienia. Rzeszów, Wydawnictwo „Otwarty rozdział”.

O żó g K., 2014 : „Współczesna polska grzeczność językowa a postmodernizm”. Kraków, Annales Universitatis Paedagogicae Cracoviensis Studia Linguistica IX. http://rep. up.krakow.pl/xmlui/bitstream/handle/11716/5095/AF167--05--Wspolczesna-polskagrzecznosc--Ozog.pdf?sequence=1\&isAllowed=y (consulté le 11 juillet 2020).

Re y A., 1989 : Le Grand Robert de la Langue française. Paris, Les Dictionnaires Robert. S c ollon R., S collon S., 1995 : Intercultural communication. A discourse approach. Oxford, Wiley-Blackwell. https://books.google.pl/books?hl=fr\&lr=\&id=rmgta7ePM $\mathrm{i} 4 \mathrm{C} \& \mathrm{oi}=$ fnd \&pg $=\mathrm{PR} 11 \& \mathrm{dq}=\mathrm{scollon}+$ intercultural + communication $\&$ ots $=$ exN0Fdap

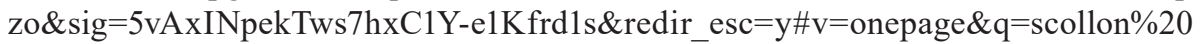
intercultural\%20communication \&f=false (consulté le 11 juillet 2020).

Tremblay O., 2015 : «L'analyse de corpus pour l'étude du lexique en classe de français ». Correspondance 1. http://correspo.ccdmd.qc.ca/index.php/document/elogede-legoportrait/lanalyse-de-corpus-pour-letude-du-lexique-en-classe-de-francais/ (consulté le 27 décembre 2019). 
Tremblay O., Anctil D., 2016 : «Les unités lexicales et leurs usages : la notion de combinatoire ». Correspondance 2. http://correspo.ccdmd.qc.ca/index.php/document/ tentative-de-rehabilitation-du-lieu-commun/les-unites-lexicales-et-leurs-usages-lanotion-de-combinatoire/\#a1 (consulté le 27 décembre 2019).

Ty ne H., 2019 : « Dan McIntyre (2018), Irony and semantic prosody revisited - compterendu de lecture par Olivier Simonin ». Langages et indentités. https://langident.hypotheses.org/275 (consulté le 27 décembre 2019).

Wierzbicka A., 1997 : Understanding Cultures Through Their Key Words: English, Russian, Polish, German, and Japanese. New York, Oxford: Oxford University Press.

Żu re k A., 2008 : „Teorie grzeczności językowej”. Kształcenie Językowe 7 [Acta Universitatis Vratislaviensis 3143], 33-44.

\section{Sitographie}

Doroszewski J., on-line: Słownik języka polskiego. PWN. https://sjp.pwn.pl/szukaj/ grzeczność.html (consulté le 27 décembre 2019).

Trésor de la langue française informatisé (TLFi). http://www.cnrtl.fr/etymologie/politesse/substantif (consulté le 27 décembre 2019). 